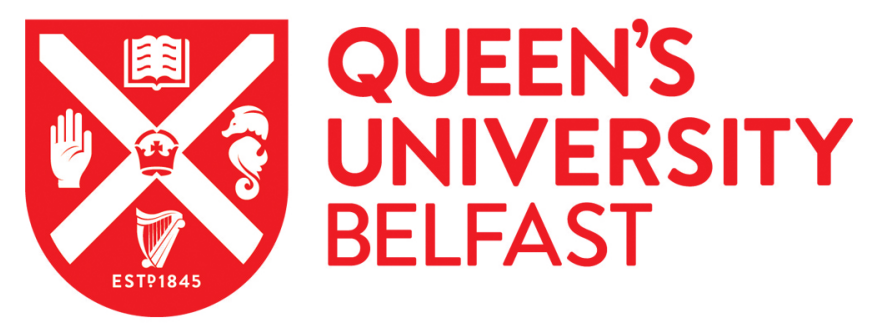

\title{
Colon and rectal cancer survival in seven high-income countries 2010- 2014: variation by age and stage at diagnosis (the ICBP SURVMARK- 2 project)
}

Araghi, M., Arnold, M., Rutherford, M. J., Guren, M. G., Cabasag, C. J., Bardot, A., Ferlay, J., Tervonen, H., Shack, L., Woods, R. R., Saint-Jacques, N., De, P., McClure, C., Engholm, G., Gavin, A. T., Morgan, E., Walsh, P. M., Jackson, C., Porter, G., ... Soerjomataram, I. (2020). Colon and rectal cancer survival in seven highincome countries 2010-2014: variation by age and stage at diagnosis (the ICBP SURVMARK-2 project). Gut. https://doi.org/10.1136/gutjnl-2020-320625

Published in:

Gut

Document Version:

Peer reviewed version

Queen's University Belfast - Research Portal:

Link to publication record in Queen's University Belfast Research Portal

Publisher rights

(c) 2020 Author(s) (or their employer(s)).

This work is made available online in accordance with the publisher's policies. Please refer to any applicable terms of use of the publisher.

\section{General rights}

Copyright for the publications made accessible via the Queen's University Belfast Research Portal is retained by the author(s) and / or other copyright owners and it is a condition of accessing these publications that users recognise and abide by the legal requirements associated with these rights.

Take down policy

The Research Portal is Queen's institutional repository that provides access to Queen's research output. Every effort has been made to ensure that content in the Research Portal does not infringe any person's rights, or applicable UK laws. If you discover content in the Research Portal that you believe breaches copyright or violates any law, please contact openaccess@qub.ac.uk. 


\section{Colon and rectal cancer survival in seven high-income countries 2010-2014: Variation by age and stage at diagnosis (the ICBP SURVMARK-2 project)}

Marzieh Araghi ${ }^{1}$, Melina Arnold ${ }^{1}$, Mark J Rutherford ${ }^{1,2}$, Marianne Grønlie Guren ${ }^{3}$, Citadel J. Cabasag ${ }^{1}$, Aude Bardot ${ }^{1}$, Jacques Ferlay ${ }^{1}$, Hanna Tervonen ${ }^{4}$, Lorraine Shack ${ }^{5}$, Ryan R Woods ${ }^{6}$, Nathalie SaintJacques $^{7}$, Prithwish De ${ }^{8}$, Carol McClure ${ }^{9}$, Gerda Engholm ${ }^{10}$, Anna Gavin ${ }^{11}$, Eileen Morgan ${ }^{1,11}$, Paul M Walsh $^{12}$, Christopher Jackson ${ }^{13}$, Geoff Porter ${ }^{14}$, Bjørn Møller ${ }^{15}$, Oliver Bucher ${ }^{16}$, Michael Eden ${ }^{17}$, Dianne L. O'Connel ${ }^{18}$ Freddie Bray ${ }^{1}$, Isabelle Soerjomataram ${ }^{1}$

${ }^{1}$ Section of Cancer Surveillance, International Agency for Research on Cancer (IARC/WHO), 150 Cours Albert Thomas, Lyon, 69372 CEDEX 08, France

${ }^{2}$ Biostatistics Research Group, Department of Health Sciences, University of Leicester, University Road, Leicester, LE1 7RH, U.K.

${ }^{3}$ Department of Oncology and K.G. Jebsen Colorectal Cancer Research Centre, Oslo University Hospital, Oslo, Norway

${ }^{4}$ Cancer Institute NSW, PO Box 41, Alexandria NSW 1435, Australia

${ }^{5}$ Cancer Control Alberta, Alberta Health Services, 2210 2nd Street, SW Calgary AB, T2S 3C3, Canada

${ }^{6}$ BC Cancer, $675 \mathrm{~W}$ 10th Ave, Vancouver, BC V5Z 1L3, Canada

${ }^{7}$ Nova Scotia Health Authority Cancer Care Program, Registry \& Analytics, 1276 South Street, Halifax, B3H 2Y9, NS, Canada

${ }^{8}$ Surveillance \& Cancer Registry, Cancer Care Ontario, 620 University Ave., Toronto, ON M5G 2L7, Canada.

${ }^{9}$ PEI Cancer Registry, 60 Riverside Dr., Charlottetown, PE C1A 8T5, Canada

${ }^{10}$ Danish Cancer Society, Strandboulevarden 49, 2100 Copenhagen, Denmark

${ }^{11}$ Northern Ireland Cancer Registry, Queen's University Belfast, Mulhouse Building, Grosvenor Road, Belfast BT12 6DP, Northern Ireland, United Kingdom

${ }^{12}$ National Cancer Registry Ireland, Cork Airport Business Park, Kinsale Road, Cork T12 CDF7, Ireland.

${ }^{13}$ Cancer Society of New Zealand, PO Box 651, Wellington 6140, New Zealand

${ }^{14}$ Canadian Partnership Against Cancer, 145 King Street West, Suite 900, Toronto, Ontario M5H 1J8, Canada

${ }^{15}$ Cancer Registry of Norway, Institute of Population-based Cancer Research, P.O. Box 5313 Majorstuen, 0304 Oslo, Norway.

${ }^{16}$ Population Oncology, CancerCare Manitoba, 675 McDermot Ave., Winnipeg, MB R3E OV9, Canada.

${ }^{17}$ National Cancer Registry and Analysis Service (NCRAS), England, the United Kingdom 
${ }^{18}$ Cancer Research Division, Cancer Council NSW, Sydney, Australia.

\section{Correspondence to:}

Isabelle Soerjomataram, MD PhD

Cancer Surveillance Section, International Agency for Research on Cancer (IARC/WHO)

150 Cours Albert Thomas, 69008 Lyon, France

soerjomatarami@iarc.fr

Word count: 4,247

Abbreviations: $\mathrm{CRC}$ - colorectal cancer; DCO - death certificate only; GP - general practitioner; ICBP - International Cancer Benchmarking Partnership; ICD-10 - International Classification of Diseases, $10^{\text {th }}$ edition; SEER - Surveillance, Epidemiology and End Results Program; TNM - Tumor, Node, Metastasis; UK - United Kingdom

Keywords: survival; colorectal cancer; international; stage at diagnosis 


\section{Abstract}

Objectives: As part of the International Cancer Benchmarking Partnership (ICBP) SURVMARK-2 project, we provide the most recent estimates of colon and rectal cancer survival in seven high-income countries by age and stage at diagnosis.

Methods: Data from 386,870 patients diagnosed during 2010-2014 from 19 cancer registries in seven countries (Australia, Canada, Denmark, Ireland, New Zealand, Norway, and the United Kingdom) were analysed. One- and five-year net survival from colon and rectal cancer was estimated by stage at diagnosis, age, and country,

Results: (One-year) and five-year net survival varied between (77.1 and 87.5\%) 59.1 and 70.9\% and (84.8 and $90.0 \%$ ) 61.6 and $70.9 \%$ for colon and rectal cancer, respectively. Survival was consistently higher in Australia, Canada and Norway, with smaller proportions of patients with metastatic disease in Canada and Australia. International differences in (one-) and five- year survival were most pronounced for regional and distant colon cancer ranging between (86.0 and 94.1\%) 62.5 and $77.5 \%$, and (40.7 and 56.4\%) 8.0 and $17.3 \%$ respectively. Similar patterns were observed for rectal cancer. Stage distribution of colon and rectal cancers by age varied across countries with marked survival differences for patients with metastatic disease and diagnosed at older ages (irrespective of stage).

Conclusions: Survival disparities for colon and rectal cancer across high-income countries are likely explained by earlier diagnosis in some countries and differences in treatment for regional and distant disease, as well as older age at diagnosis. Differences in cancer registration practice and different staging systems across countries may have impacted the comparisons. 


\section{Summary Box}

\section{What is already known about this subject?}

- Survival from colon and rectal cancer shows substantial geographical variation and differences in outcomes exist even across high-income countries. Stage and age at diagnosis remain the key prognostic factors, which we explore in-depths in this international population-based study.

\section{What are the new findings?}

- Based on up-to-date data from the high quality population-based cancer registries in Australia, Canada, Denmark, Ireland, New Zealand, Norway, and the United Kingdom (UK), this study assesses short-term and long-term (1- and 5-year) survival of colorectal cancer cases diagnosed between 2010-2014 by age and stage.

- Differences in stage distribution of colon and rectal cancers were observed, with large proportions of cases with localized colon or rectal cancers in Norway and Australia (also the UK for colon cancer) and small proportions with metastatic cancers in Australia and Canada (also Ireland for rectal cancer).

- While survival differences across countries were evident for all stage groups, large variation was observed for regional and advanced disease.

\section{How might it impact on clinical practice in the foreseeable future?}

- Our results suggest that survival disparities across countries could partly be explained by differences in treatment and management of regional and distant colon and rectal cancers, and by earlier diagnosis in some countries.

- Future research into the role of screening programmes as well as into factors influencing treatment decision making by countries is warranted to identify the drivers behind the observed survival differences. 


\section{Introduction}

Colon and rectal cancers (CRC) were the third most common cancer (1.8 million cases) and the second most common cause of cancer-related death $(881,000$ deaths) for both men and women worldwide in 2018.[1] Most of this burden is concentrated in high and very high-income countries, where incidence is high and the prospects of cure are considerably better than in other regions of the world.[2] Yet, marked survival differences have long existed across high-income countries[3]. In an effort to drive change, the International Cancer Benchmarking Partnership (ICBP) brings together clinicians, policy-makers, researchers, and cancer data experts seeking to explain cancer survival differences between high-income countries with similar health systems e.g. similar healthcare expenditure, universal healthcare coverage and population coverage through cancer registries[3].

In previous analyses of CRC survival for patients diagnosed in 2000-2007, one-year net survival from colon cancer ranged between $80.2 \%$ in Australia and $67.4 \%$ in the United Kingdom (UK), whereas for rectal cancer it was highest in Sweden (84.4\%) and lowest in the UK (75.2\%).[4] For both cancers, betweencountry differences in net survival were largest for the oldest age groups and for patients with more advanced stage of disease at diagnosis. For example, one-year net survival for colon cancer patients with 'localized' stage ranged between $95.1 \%$ in Canada and $91.3 \%$ in the United Kingdom, compared with 'distant' stage ranging between $42.0 \%$ in Australia and $34.2 \%$ in the UK. Differences in uptake and coverage of new treatment advances such as improved surgical techniques,[5] adjuvant chemotherapy,[6] pre-operative radiotherapy,[7] or the use of palliative chemotherapy[8] and multi-modal treatment approaches for resectable metastases[9] might explain some of these survival differences. In addition, differences in time (delays) to diagnosis and in access to cancer care (from primary healthcare) may partly contribute to the observed survival variation.[10] Monitoring survival by stage at diagnosis remains vital to identify drivers of overall differences and to assess the effectiveness of national health systems.

In this study and as part of the ICBP SURVMARK-2 project[3], we provide the most recent estimates of colon and rectal cancer survival by age and stage at diagnosis, using population-based data from 19 cancer registries in seven countries (Australia, Canada, Denmark, Ireland, New Zealand, Norway, and the UK). We compare distributions of stage at diagnosis and examine overall and stage-specific survival at one and five years after diagnosis. 


\section{Methods}

\section{Data sources}

During the course of the ICBP SURVMARK-2 project, data for patients diagnosed with CRC were collected from 21 population-based cancer registries in seven countries. Data submitted included information on histology, morphology, basis of diagnosis, stage at diagnosis and treatment. A number of quality control measures were carried out on each dataset. This included screening data for specific anomalies including instances of negative survival duration, out-of-range dates of diagnosis and/or dates of death, availability of stage at diagnosis information and invalid vital status codes. Cases were selected and coded according to the following ICD-10 rubrics:[11] colon (C18-19), and rectum (C20) including all morphologies. In the current analyses, we included patients diagnosed during 2010-2014 from the following 19 registries that provided sufficient information on stage at diagnosis ( $\geq 50 \%$ of cases with known stage, Supplementary Tables 1A \& 1B): Australia (New South Wales, Victoria), Canada (Alberta, British Columbia, Manitoba, New Brunswick, Newfoundland, Nova Scotia, Ontario, Prince Edward Island, Saskatchewan), Ireland (2010-2013), Denmark, New Zealand, Norway and the UK (England, Scotland, Wales, Northern Ireland). Out of 405,255 colon and rectal cancer patients (colon cancer: 294,996; rectal cancer: 110,259) diagnosed during 2010-2014, we excluded cases diagnosed based on death certificate only (DCO) or at autopsy $(\mathrm{N}=4,613,1.1 \%)$, below the age of 15 or above age 99 at diagnosis $(\mathrm{N}=448,0.1 \%)$, with inconsistencies in stage information $(\mathrm{N}=1,302,0.3 \%)$, with in situ tumours $(\mathrm{N}=373,0.1 \%)$, and second or higher sequenced cancers diagnosed at the same site $(\mathrm{N}=11,649,2.9 \%)$. Using these criteria, a total of $386,870$ (95.5\% of those eligible) (colon cancer: 280,251 ; rectal cancer: 106,619$)$ patients diagnosed during 2010-2014, were included in the analyses.

Each participating cancer registry provided information on pre-treatment pathological and clinical T, N, M records, grouped TNM stage, SEER summary stage 2000 (SEER SS2000) and/or Duke's stage (Supplementary Figure 1A). A previously developed algorithm[12] was used to translate both grouped TNM and/or Dukes' as well as individual T, N, M elements to SEER Summary Staging (categorized into localized, regional, distant and missing), enabling us to include all seven countries in comparative analyses. A flowchart of how registry-specific staging information was mapped to SEER staging is available in Supplementary Figures IA \& IB. All analyses were carried out using grouped TNM where possible and mapped SEER stage for all countries and jurisdictions. We present survival estimates for colon and rectal cancers separately, for all ages combined and four age groups at diagnosis: 15-49, 50-64, 65-79, and 80-99 years. For simplicity, we used stages I-IV when referring to TNM stage, and 'localized', 'regional', and 'distant' when referring to SEER SS2000. 


\section{Statistical analyses}

For cases with missing stage at diagnosis, stage information was imputed using the multiple imputation (mi) command with the following covariates: sex, age, year of diagnosis, survival time, and the NelsonAalen estimator of the cumulative hazard. We ran the imputation procedure 30 times and combined the results using Rubin's rules to estimate net survival and 95\% confidence interval (CI).[13]

We reported net survival with accompanying 95\% CIs, which is the probability of survival for cancer patients in a hypothetical situation where cancer is considered the only possible cause of death. Background mortality in the general population of each jurisdiction was obtained from lifetables of all-cause death rates during 1995-2014 by sex, single year of age and calendar year for the respective study period. Follow-up was available until Dec 31, 2015, for all patients except for those in Ontario, where follow-up was limited to Dec 31, 2014. Net survival estimates at one and five years after diagnosis were computed by age, sex, stage at diagnosis, and cancer site for each jurisdiction and for the Canadian, Australian and UK registries combined using Pohar Perme estimators,[14] which has been shown to be an unbiased method to estimate cancer-specific survival.[15] We used the period approach for patients diagnosed in 2010-14 (period window: 2012-14) which has been shown to perform particularly well in the prediction of up-to-date cancer survival.[16] Age-standardization was carried out using the International Cancer Survival Standard (ICSS) weights.[17] While in the main manuscript we report stage-specific survival estimates by stage at diagnosis after imputation, we also present results based on original, non-imputed, stage categories in Supplementary tables. 


\section{Patient and Public Involvement}

The International Cancer Benchmarking Partnership is a multi-partner collaboration that involves clinicians, policy-makers, researchers, and cancer data experts. While patients were not directly involved in the analytical phase of the study, we will incorporate survival estimates into a publicly available website (http://gco.iarc.fr/survival/survmark/) to support dissemination of findings to patient and public via simple user-generated and automatic graph layouts.

\section{Results}

Age at diagnosis and cancer stage by country

The median age and distribution of cases by cancer site, country and stage at diagnosis for TNM stage and mapped SEER stage are given in Table 1. For both colon and rectal cancers, the median age at diagnosis was slightly higher in New Zealand, Norway and the UK when compared with Australia, Denmark, Ireland and Canada. Among jurisdictions included in this study, the proportion of colon cancer patients with missing stage at diagnosis was highest in the UK (TNM: 39.5\%; SEER: 37.2\%) and lowest in Canada (TNM: 7.5\%; SEER: 6.4\%). For rectal cancer, a similar pattern was seen i.e. proportion with missing TNM stage was $39.4 \%$ (SEER: $37.0 \%$ ) in the UK and 10.8\% (SEER: 6.8\%) in Canada. Multiple imputation did not substantially alter the distribution of stage for colon or rectal cancer (Table 1).

The distribution of stage at diagnosis (localized, regional and distant) also varied across countries (Table 1, Figure 1). For example, the proportion of patients with 'distant' stage colon cancer was lowest in Australia (21.5\%) and Canada (22.8\%) and highest in the UK (28.6\%), followed by New Zealand (28.2\%). For rectal cancer, the proportion of patients diagnosed with 'distant' stage was lowest in Australia (17.7\%) and highest in New Zealand (25.8\%).

One-year and five-year net survival by country

Overall, one-year age-standardized net survival from colon cancer ranged from $77.1 \%$ in the UK to $87.5 \%$ in Australia compared to 80.0-84.2\% elsewhere (Supplementary Table 2A). One-year age-standardized net survival from rectal cancer ranged from $84.8 \%$ in the UK to $90.0 \%$ in Australia compared to $85.9-89.1 \%$ elsewhere (Supplementary Table 3A). For both colon and rectal cancers, similar patterns of net survival across countries were observed five years after diagnosis (Supplementary Tables 2B \& 3B). Five-year survival from colon cancer ranged from 59.1\% in the UK to $70.9 \%$ in Australia (Supplementary Table 2B). 


\begin{tabular}{|c|c|c|c|c|c|c|c|c|c|c|c|c|c|c|c|c|}
\hline \multirow[b]{3}{*}{ Country } & \multicolumn{8}{|c|}{ Colon cancer } & \multicolumn{7}{|c|}{ Rectal cancer } & \multirow{3}{*}{$\begin{array}{c}\text { Imputed } \\
\mathbf{( \% )}\end{array}$} \\
\hline & \multicolumn{3}{|c|}{ TNM stage } & \multirow[b]{2}{*}{$\begin{array}{c}\text { Imputed } \\
(\%)\end{array}$} & \multicolumn{4}{|c|}{ Mapped SEER } & \multicolumn{3}{|c|}{ TNM stage } & \multirow[b]{2}{*}{$\begin{array}{c}\text { Imputed } \\
(\%)\end{array}$} & \multicolumn{3}{|c|}{ Mapped SEER } & \\
\hline & Stage & $\begin{array}{c}\text { Median } \\
\text { age (IQR) }\end{array}$ & $\begin{array}{l}\text { Number of } \\
\text { cases (\%) }\end{array}$ & & Stage & $\begin{array}{c}\text { Median age } \\
\text { (IQR) }\end{array}$ & $\begin{array}{l}\text { Number of } \\
\text { cases (\%) }\end{array}$ & $\begin{array}{c}\text { Imputed } \\
(\%)\end{array}$ & Stage & $\begin{array}{c}\text { Median age } \\
\text { (IQR) }\end{array}$ & $\begin{array}{l}\text { Number of } \\
\text { cases (\%) }\end{array}$ & & Stage & $\begin{array}{c}\text { Median age } \\
\text { (IQR) }\end{array}$ & $\begin{array}{l}\text { Number of } \\
\text { cases (\%) }\end{array}$ & \\
\hline \multirow{6}{*}{ Australia $^{\circ}$} & All & $72(62-80)$ & 12,932 & & All & $72(62-80)$ & 30,802 & & All & $67(57-77)$ & 4,877 & & All & $67(58-76)$ & 11,058 & \\
\hline & I & $70(62-79)$ & $2455(21.2)$ & 20.8 & Localised & $72(63-80)$ & $10743(38.0)$ & 37.7 & I & $67(58-76)$ & $1360(35.0)$ & 34.8 & Localised & $67(59-76)$ & $4173(44.4)$ & 45.0 \\
\hline & II & $74(65-81)$ & $3684(31.8)$ & 31.7 & Regional & $72(63-80)$ & 11477 (40.6) & 40.8 & II & $69(60-77)$ & 847 (21.8) & 21.0 & Regional & $66(57-76)$ & 3595 (38.3) & 37.3 \\
\hline & III & $71(61-79)$ & 3076 (26.6) & 26.4 & Distant & $70(60-79)$ & $6028(21.3)$ & 21.5 & III & $65(56-76)$ & $1009(26.0)$ & 25.9 & & $66(57-76)$ & $1629(17.3)$ & 177 \\
\hline & IV & $69(59-78)$ & $2364(20.4)$ & 21.1 & Distant & & & 21.5 & IV & $65(56-75)$ & $665(17.1)$ & 18.3 & Distant & & & \\
\hline & Missing & $76(63-85)$ & $1353(10.5)$ & & Missing & $74(63-84)$ & $2554(8.3)$ & & Missing & $70(58-81)$ & 996 (20.4) & & Missing & $68(57-79)$ & $1661(15.0)$ & \\
\hline \multirow{5}{*}{$\begin{array}{l}\text { Canadian } \\
\text { provinces }^{\dagger}\end{array}$} & All & $71(61-80)$ & 58,749 & & All & $71(61-80)$ & 58,749 & & All & $66(57-76)$ & 20,271 & & All & $66(57-76)$ & 20,271 & \\
\hline & I & $70(61-79)$ & $11842(21.8)$ & 22.3 & Localised & $71(62-80)$ & $20435(37.2)$ & 38.0 & I & $67(58-76)$ & $4637(25.6)$ & 25.3 & Localised & $67(58-76)$ & 6917 (36.6) & 36.3 \\
\hline & II & $73(63-81)$ & $15414(28.4)$ & 27.9 & Regional & $71(61-80)$ & $21934(39.9)$ & 39.2 & II & $68(59-77)$ & $3742(20.7)$ & 19.6 & Regional & $65(57-75)$ & $8333(44.1)$ & 43.6 \\
\hline & III & $70(61-79)$ & $15161(27.9)$ & 27.8 & Distant & $69(60-79)$ & $12592(22.9)$ & 22.8 & III & $65(56-74)$ & $6327(35.0)$ & 35.7 & Distant & $65(56-75)$ & $3637(19.3)$ & 20.0 \\
\hline & $\begin{array}{c}\text { IV } \\
\text { Missing }\end{array}$ & $\begin{array}{l}69(60-79) \\
76(63-85)\end{array}$ & $\begin{array}{c}11912(21.9) \\
4420(75)\end{array}$ & 21.9 & $\begin{array}{l}\text { Distant } \\
\text { Missing }\end{array}$ & $76(64-86)$ & $3788(6.4)$ & & $\begin{array}{l}\text { IV } \\
\text { Missing }\end{array}$ & $\begin{array}{l}66(56-75) \\
68(56-81)\end{array}$ & $\begin{array}{l}3372(18.7) \\
2193(1088)\end{array}$ & 19.4 & $\begin{array}{l}\text { Distant } \\
\text { Missing }\end{array}$ & $70(57-82)$ & $1384(6.8)$ & \\
\hline \multirow{6}{*}{ Denmark } & All & $72(65-79)$ & 14,690 & & All & $72(65-79)$ & 14,690 & & All & $69(62-77)$ & 7,584 & & All & $69(62-77)$ & 7,584 & \\
\hline & I & $71(64-78)$ & 1561 (12.6) & 14.0 & Localised & $72(65-79)$ & $4799(38.8)$ & 40.8 & I & $69(63-76)$ & $1148(18.2)$ & 19.9 & Localised & $69(63-76)$ & 2499 (39.6) & 42.1 \\
\hline & II & $73(66-80)$ & $4093(33.1)$ & 34.2 & Regional & $71(64-79)$ & $4058(32.8)$ & 32.8 & II & $70(63-77)$ & $1649(26.1)$ & 26.9 & Regional & $68(61-76)$ & $2360(37.4)$ & 35.9 \\
\hline & III & $71(63-78)$ & $3196(25.9)$ & 25.4 & Distant & $71(63-78)$ & $3500(28.3)$ & 26.4 & III & $68(61-75)$ & $2060(32.7)$ & 31.3 & Distant & $69(62-76)$ & $1452(23.0)$ & 21.9 \\
\hline & IV & $71(63-78)$ & $3500(28.3)$ & 26.5 & & & & & & $69(62-76)$ & $1452(23.0)$ & 21.9 & & & & \\
\hline & Missing & $75(67-83)$ & $2340(15.9)$ & & Missing & $75(67-83)$ & $2333(15.9)$ & & Missing & $72(64-81)$ & $1275(16.8)$ & & Missing & $72(64-81)$ & $1273(16.8)$ & \\
\hline \multirow[t]{5}{*}{ Ireland" } & All & $71(62-79)$ & 6,863 & & All & $71(62-79)$ & 6,863 & & All & $67(59-76)$ & 2,637 & & All & $67(59-76)$ & 2,637 & \\
\hline & I & $70(62-78)$ & $897(14.3)$ & 14.3 & Localised & $71(64-79)$ & $2304(36.8)$ & 37.0 & I & $69(61-77)$ & $437(18.5)$ & 18.6 & Localised & $69(62-77)$ & $857(36.2)$ & 35.7 \\
\hline & II & $73(64-80)$ & $1905(30.5)$ & 30.8 & Regional & $70(62-78)$ & $2360(37.7)$ & 37.5 & II & $70(63-77)$ & $502(21.2)$ & 20.6 & Regional & $66(57-75)$ & $1047(44.3)$ & 45.3 \\
\hline & III & $70(61-78)$ & $1859(29.7)$ & 29.4 & Distant & $71(61-78)$ & $1593(25.5)$ & 25.5 & III & $65(57-74)$ & $965(40.8)$ & 41.7 & Distant & $66(57-75)$ & $462(19.5)$ & 19.1 \\
\hline & $\begin{array}{l}\text { IV } \\
\text { Missing }\end{array}$ & $\begin{array}{l}71(61-78) \\
75(62-84)\end{array}$ & $\begin{array}{c}1593(25.5) \\
609(8.9)\end{array}$ & 25.5 & $\begin{array}{l}\text { Distant } \\
\text { Missing }\end{array}$ & $75(62-84)$ & $606(8.8)$ & & $\begin{array}{l}\text { IV } \\
\text { Missing }\end{array}$ & $\begin{array}{l}66(57-75) \\
69(59-78)\end{array}$ & $\begin{array}{l}462(19.5) \\
271(10.3)\end{array}$ & 19.1 & Missing & $69(59-78)$ & $271(10.3)$ & \\
\hline \multirow[t]{5}{*}{$\begin{array}{l}\text { New } \\
\text { Zealand }\end{array}$} & & & & & All & $73(64-81)$ & 11,049 & & & & & & All & $69(60-77)$ & 3,811 & \\
\hline & & & & & Localised & $73(65-79)$ & 2644 (26.8) & 25.4 & & & & & Localised & $70(61-78)$ & 667 (31.2) & 30.5 \\
\hline & & & & & Regional & $73(65-80)$ & 4581 (46.4) & 46.5 & & & & & Regional & $69(61-78)$ & 888 (41.6) & 43.7 \\
\hline & & & & & Distant & $71(62-79)$ & 2641 (26.8) & 28.2 & & & & & Distant & $68(58-77)$ & $581(27.2)$ & 25.8 \\
\hline & & & & & Missing & $78(69-85)$ & $1183(10.7)$ & & & & & & Missing & $68(58-77)$ & $1675(44.0)$ & \\
\hline \multirow[t]{5}{*}{ Norway } & All & $73(64-81)$ & 13,875 & & All & $73(64-81)$ & 13,875 & & All & $69(61-78)$ & 5,334 & & All & $73(64-81)$ & 5,334 & \\
\hline & I & $74(65-80)$ & $1734(13.9)$ & 14.3 & Localised & $74(65-81)$ & $5519(44.1)$ & 43.7 & I & $69(62-77)$ & $1130(29.1)$ & 29.2 & Localised & $70(62-78)$ & $1972(50.8)$ & 51.0 \\
\hline & II & $73(66-81)$ & $4279(34.2)$ & 33.5 & Regional & $72(64-80)$ & 3568 (28.5) & 28.3 & II & $70(62-78)$ & $913(23.5)$ & 24.1 & Regional & $69(61-78)$ & 918 (23.6) & 24.3 \\
\hline & III & $72(64-80)$ & 3074 (24.6) & 24.2 & Distant & & & 28.0 & III & 69 (61-78) & 847 (21.8) & 22.1 & Distant & $70(60-78)$ & $992(25.6)$ & 24.7 \\
\hline & $\begin{array}{l}\text { IV } \\
\text { Missing }\end{array}$ & $\begin{array}{l}71(63-80) \\
77(67-85)\end{array}$ & $\begin{array}{c}3414(27.3) \\
1374(9.9)\end{array}$ & 27.9 & $\begin{array}{l}\text { Distant } \\
\text { Missing }\end{array}$ & $77(67-85)$ & $\begin{array}{c}3414(2 / .3) \\
1374(9.9)\end{array}$ & 28.0 & $\begin{array}{l}\text { IV } \\
\text { Missing }\end{array}$ & $\begin{array}{l}67(58-76) \\
70(61-78)\end{array}$ & $\begin{array}{c}992(25.6) \\
1452(27.2)\end{array}$ & 24.6 & Missing & 70 (61-78) & $1452(27.2)$ & 24.1 \\
\hline \multirow[t]{6}{*}{$\begin{array}{l}\text { UK } \\
\text { registries }^{\ddagger}\end{array}$} & All & $73(64-81)$ & 144,223 & & All & $73(64-81)$ & 144,223 & & All & 70 (61-78) & 55,924 & & All & 70 (61-78) & 55,924 & \\
\hline & I & $70(63-78)$ & $13194(15.1)$ & 14.4 & Localised & $72(64-79)$ & $35982(39.8)$ & 37.7 & I & $69(62-77)$ & $8300(24.5)$ & 23.8 & Localised & 70 (62-78) & $\begin{array}{l}15108 \\
(42.9)\end{array}$ & 41.5 \\
\hline & II & $73(65-81)$ & $25798(29.6)$ & 28.9 & Regional & $72(63-80)$ & $30866(34.1)$ & 33.6 & II & $71(62-78)$ & $6859(20.2)$ & 19.8 & Regional & $68(60-76)$ & $\begin{array}{l}13343 \\
(37.9)\end{array}$ & 38.0 \\
\hline & III & $71(63-79)$ & $23927(27.4)$ & 26.9 & Distant & $73(63-81)$ & $23666(26.1)$ & 28.6 & III & $68(59-76)$ & $\begin{array}{l}11776 \\
(34.7)\end{array}$ & 35.0 & Distant & $70(60-78)$ & 6785 (19.3) & 20.5 \\
\hline & IV & $73(63-81)$ & 24289 (27.9) & 29.7 & & & & & IV & 70 (60-78) & $6954(20.5)$ & 21.4 & & & & \\
\hline & Missing & $74(65-82)$ & $57015(39.5)$ & & Missing & $74(65-82)$ & $53709(37.2)$ & & Missing & $71(62-80)$ & $\begin{array}{l}22035 \\
(39.4)\end{array}$ & & Missing & $71(62-80)$ & $\begin{array}{l}20688 \\
(37.0)\end{array}$ & \\
\hline
\end{tabular}

${ }^{\dagger}$ Canadian provinces included: Alberta, British Columbia, New Brunswick, Manitoba, Newfoundland, Nova Scotia, Ontario, Prince Edward Island, and

${ }^{\ddagger}$ United Kingdom registries included: England, Northern Ireland, Scotland, and Wales

- Australia registries included: Victoria (Data on both TNM and SEER) and New South Wales (Data on SEER only), Ireland (2010-2013)

Table 1. Median age at diagnosis, number of cases and imputed proportion (\%) of colon and rectal cancers by stage and country, 2010-2014 
Five-year survival from rectal cancer ranged from $61.6 \%$ in Ireland to $70.9 \%$ in Canada (Supplementary Table 3B).

One- and five-year net survival by age and country

One- and five-year net survival from both cancers decreased with increasing age at diagnosis while international differences in survival were largest for the oldest patient groups (80-99 years). For example, one-year survival for colon cancer patients aged 15-49 years ranged between 84.8\% (New Zealand) and 92.0\% (Australia) while survival for patients aged 80-99 years ranged between 57.7\% (UK) and 76.9\% (Australia) (Supplementary Table 2A). Similar age patterns were also seen for rectal cancer, for example one-year survival for rectal cancer patients aged 15-49 years ranged between 90.4\% (New Zealand) and 95.9\% (Australia), and 69.0\% (Ireland) and 80.2\% (Australia) for patients aged 80-99 years (Supplementary Table 3A).

One- and five-year net survival by stage and country

International differences in age-standardized net survival were evident, in particular for patients with regional/Stage III and distant/Stage IV colon and rectal cancers (Figures $2 \&$ 3). For example, using SEER stage, one-year survival for colon cancer patients with 'localized' disease ranged between $96.0 \%$ (Canada/UK) and $98.3 \%$ (New Zealand), whereas for 'regional' colon cancer it ranged between $86.0 \%$ (UK) and 94.1\% (Australia), and for 'distant' stage it ranged between 40.7\% (United Kingdom) and 56.4\% (Australia) (Supplementary Table 2A, Figure 3). Patterns were similar for survival for colon cancer patients five years after diagnosis (Supplementary Table 2B, Figure 3). For rectal cancer, the international differences in net survival across stage also varied by country, with similar patterns as those observed for colon cancer (Supplementary Tables 3A \& 3B, and Figures $2 \& 3$ ).

One- and five-year net survival by age, stage and country

Overall, international differences in one- and five-year net survival from both colon and rectal cancer were more pronounced with increasing age and especially for those with regional and advanced stage of disease (Figures 4-7). One- and five-year survival for the oldest colon cancer patients with 'distant' stage ranged between 17.7\% (Ireland) and 30.2\% (Denmark), and 1.3\% (UK) and 8.3\% (Denmark), respectively (Supplementary Tables 2A and 2B). One- and five-year survival for rectal cancer patients with 'distant' stage aged 80-99 years ranged between $29.0 \%$ (Ireland) and 37.7\% (Denmark), and 1.1\% (UK) and 7.2\% (Denmark), respectively (Supplementary Tables 3A and 3B). Figures 4-7 also present the proportion of patients by stage category across age groups, for example the proportion of colon cancer patients with 'distant' stage among those aged 80-99 years was highest in the UK (32.5\%) and between $20.8 \%$ and $28.1 \%$ 
elsewhere, whereas the proportion of rectal cancer patients with 'distant' stage in this oldest age group was highest in New Zealand (28.7\%) and between 18.7\% and 25.0\% elsewhere (Figures 6 \& 7, and Supplementary Figures $2 \& 3$ ).

\section{Supplementary analyses}

One-year net survival from colon and rectal cancers was similar for males and females (Supplementary Tables 4A, 5A, 6A, 7A), however, five-year survival was higher for females (Supplementary Tables 4B, 5B, 6B, 7B). Stage-specific survival estimates including the missing category (without multiple imputation) were slightly higher compared with survival estimates after multiple imputation. For example, in Canada (with the lowest proportion of missing stage) one-year survival for imputed distant stage colon cancer was $50.0 \%$ and the estimate without imputation was $51.0 \%$. In the UK, where the proportion of those with missing stage information was highest, the respective estimates were $40.7 \%$ and $42.9 \%$ (Supplementary Table 8A, Figure 4A). This is due to the somewhat poorer survival for patients with missing stage. For example, among colon cancer patients for whom SEER stage was missing, the one-year net survival ranged between $68.5 \%$ and $81.6 \%$ versus $77.1 \%$ and $87.5 \%$ for overall colon cancer cases, and $74.3 \%$ and $89.3 \%$ versus $84.8 \%$ and $90.0 \%$ for rectal cancer, respectively (Supplementary Tables $8 \mathrm{~A} \&$ 9A, Figures $4 \& 5$ ).

As for specific results by jurisdiction within country, variation in stage distribution was evident; in the UK, the proportions of cases with localized colon and rectal cancers were largest in Scotland $(42.0 \%$ and $49.9 \%$, respectively - Supplementary Table 10). Net survival estimates for rectal cancer were generally better in Scotland (e.g. at 5 years for localized disease $88.2 \%$ versus $80.3 \%$ in Wales), and for colon cancer in Northern Ireland (e.g. at 5 years for localized disease $95.2 \%$ versus $83.1 \%$ in Wales - Supplementary Tables 11-14). In Canada, the proportion with localized colon cancer varied from $32.2 \%$ to $45.7 \%$ and $29.1 \%$ to $45.4 \%$ for rectal cancer (Supplementary Table 15). Survival also varied; for example for the larger Canadian provinces 5-year net survival from localized colon cancer ranged between $86.4 \%$ (Saskatchewan) and 96.2\% (New Brunswick, Supplementary Tables 16-19). Similarly, we also observed differences in stage distribution in Australia e.g. 31.3\% were localized colon cancer in New South Wales and 46.8\% in Victoria (Supplementary Table 20, while 5-year net survival was approximately 95\% in both jurisdictions (Supplementary Tables 21-24). As previously observed, survival differences within countries were largely driven by variations in survival among the older cancer patients and those with advanced disease. 


\section{Discussion}

The current study has shown survival differences for colon and rectal cancers by age and stage at diagnosis across seven high-income countries with similar health systems. For colon cancer, age-standardized fiveyear net survival from colon and rectal cancers ranged between $59.1 \%$ and $70.9 \%$ and $61.6 \%$ and $70.9 \%$, respectively, and tended to be higher in Australia and Canada, intermediate in Denmark and Norway and lower in Ireland, New Zealand and the UK. Stage at diagnosis varied by countries; with large proportions with localized colon and rectal cancers in Norway and Australia (as well as the UK for colon cancer) and small proportions with metastatic cancers in Australia and Canada (also Ireland for rectal cancer). Survival differences persisted within each stage at diagnosis and were most pronounced for regional and distant disease as well as with increasing age at diagnosis. Compared with the first phase of ICBP,[4] survival improvements are evident in particular for metastatic disease. For example in Denmark, 1-year survival of metastatic colon and rectal cancer improved by 14 percentage point (40.7\% to $54.4 \%)$ and 11 percentage point (52.4\% to $63.4 \%$ ), respectively. A study of CRC cases in the U.S. [18] showed that 5-year relative survival (colon cancer: 64.4\%, rectal cancer: 66.6\%) are closer our estimates for Denmark and Norway. Direct comparison between our study and their estimate needs to also consider differences in various factors including diagnostic and treatment practices as well as access to health care system.[19]

Stage at diagnosis is an important determinant of survival and partly explains international differences in survival. Generally we observed smaller proportions of patients with metastatic disease in Australia and Canada, and larger proportions with localized disease in Australia and Norway. The distribution of stage at diagnosis maybe affected by national early detection and screening programs as well as by country-specific diagnostic pathways and clinical procedures. Gradual implementation of the CRC screening program started in the mid-2006s (UK), 2006-2020 (Australia, roll-out with additional age groups added each year), 2007-2012 (Canada), 2012 (Ireland and Norway [the latter started with a pilot program, national program in 2019]), 2014 (Denmark)[20] and in 2017 in New Zealand.[21] Therefore, the impact of screening activities on stage distributions during the time period covered by this study (2010-2014) is limited to the UK where screening started more than a decade ago. Comparison between country needs to take into account screening uptake e.g. in the case of the UK 52\%, [20] and also case mix that follows in populations with screening programme. Screening for colon and rectal cancers typically leads to an initial increase in incidence attributable to a greater detection of, and shift towards, early-stage cancers, followed by decreases in incidence due to removal of premalignant adenomas.[22] Screening programs for CRC have furthermore been associated with a reduction in colon cancer cases diagnosed as an emergency.[23] Continuous monitoring and quality assurance of early detection and screening programs and detailed assessment of their impact on survival are therefore warranted.[24] 
Another phenomenon that has been proposed to explain differences in international stage distributions and stage-specific survival is stage migration.[25] Thoroughness of examination to determine stage in patients may differ between countries. For example, a study comparing the number of lymph nodes examinations among cancer patients across European countries showed that lower number of lymph nodes examinations led to misclassification of advanced stage cancers (towards early stage), resulting in lower survival in both early and advanced stage categories.[26] International differences in diagnostic guidelines, access to early detection, and adherence to protocols could potentially bias stage-specific survival comparisons across countries. In addition, variations in clinical and pathological practice with regards to clinical examinations, nodal assessment and the use of imaging technology to detect small lymph nodes or distant metastases may have contributed to differences in the composition of patients within a specific stage grouping.[27] It is therefore important to interpret findings from our study in light of local clinical practice, and to ensure that registries have data collection protocols that are as uniform as possible.[28]

Another potential factor that may influence early detection is patients' behaviours towards symptoms, as these have been linked to diagnostic delays and can impact the time from the first symptoms to diagnosis, as well as time from diagnosis to staging.[29] For example, in the UK, the general population often report 'embarrassment' as the main barrier to going to the doctor when a symptom might be serious.[30] Improved interventions to address barriers to early presentation and increase confidence to approach primary care physicians (particularly for older patients) may potentially reduce delays in diagnosis and ultimately improve survival. Furthermore, changes in regional and national health care policies can influence patients' pathways. For example, urgent referrals for cancer investigation have been implemented in Denmark and this has been shown to reduce diagnostic and treatment delays.[31] The use of urgent referrals by general practitioners (GPs) has also proven efficient in improving cancer prognosis.[32] A better understanding of patients' symptoms when presenting to GPs may result in more rapid and accurate diagnosis that will lead to a more efficient diagnostic pathway.[33] Finally success in implementation of health care policies largely depend on contexts of the local setting and its health system and therefore tailoring of strategies is key to ensure effective policy.

The existing international variation in survival from CRC could also in part be attributable to national differences in treatment practices, in particular the receipt of surgery and chemotherapy. Surgical resection is widely accepted as standard treatment for localized and regional stages of colon and rectal cancers. Yet, the receipt of surgical treatment varies by country, age and stage. For example, the proportion of colon cancer patients receiving surgical treatment ranged from 68.4\% in England to 81.3\% in Sweden, and from $59.9 \%$ to $70.8 \%$ for rectal cancer, respectively.[34] The variation is even larger for CRC patients older than 75 years; for example for colon cancer patients in England this was $59.7 \%$ as compared to $80.9 \%$ in 
Sweden.[34] In addition to surgery, systemic therapy is an important treatment modality for regional CRC.[35] Studies have shown large variations in the use of adjuvant chemotherapy [36] and pre-operative radiotherapy [37] across countries. For example 56\% of cases with Dukes' stage C colon cancers in the United States received chemotherapy while only $42 \%$ of cases with the same stage received chemotherapy in Northern Europe [38]. These disparities are in general greater for the oldest patient group.[39] Patients with resectable metastases may benefit from multi-disciplinary treatment with surgery and chemotherapy; while beneficial effects of chemotherapy - with/without palliative surgery of the primary tumour - have been reported for patients with wide-spread metastases.[9] Treatment harmonisation between countries in line with recent clinical guidelines should decrease the international survival gap.

When interpreting the study results, differences in registration practice and staging systems need to be considered. As part of the ICBP SURVMARK-2 study protocol, data quality checks using standard indicators were carried out and variables were harmonized in close collaboration with participating cancer registries.[40, 41] The overall data quality was high, with fewer than $1.1 \%$ of cases registered as DCO, yet differences in data handling and registration practice may still have partially biased the survival comparisons. For example, problematic death linkages may contribute to missing deaths and overestimated survival. To put this in context however, a recent study showed that even under the extreme scenarios for incorrect registration, for example, recording the date of cancer recurrence instead of the date of primary cancer diagnosis, very little of the international differences in survival could be explained by differences in cancer registration.[42] Another study suggested incompleteness of case ascertainment may induce an error in survival time (survival time would be too short due to processing information from death certificates, especially for fatal cancers) by a magnitude of $<1.9 \%$ for the patients diagnosed with CRC in England.[43]

Data on stage was provided using different classification systems, which required the conversion and mapping of different stage variables to one common classification. The TNM system remains the preferred staging classification, however for the sake of comparison in this study all cases were mapped to the SEER SS2000 system using previously defined algorithms.[12] Due to inconsistencies in the staging of certain tumour types across staging systems, this process might have resulted in stage misclassification.[44] A previous study showed that transformation of the Duke's system to TNM led to $10 \%$ of stage IV colon cancers being misclassified as stage III.[44] In this study the Duke's system (with or without integrated staging) was used only in the UK (except in England). The staging distribution for Scotland, which uses the Duke's staging system, was shown to be similar to that for England where only integrated staging was used (stage I, II, III \& IV were 16\%, 29\%, 26\%, 29\% in Scotland and 14\%, 29\%, 27\%, 30\% in England). Differences in the timing of stage data collection processes across registries may, for example, affect staging of rectal cancer patients who have undergone preoperative radio- or chemo- therapy, which can lead to 
reductions in the tumour size or the number of involved lymph nodes.[37]. Although the data collection protocol specified collection of pre-treatment stage data, stage comparisons (and survival by stage) need to be interpreted with caution and future work should focus on improvements in this area.[45] Routine collection of information on diagnostic procedures performed to define stage, such as pathological examination of lymph nodes or clinical assessment using imaging for distant metastasis should be considered. In collaboration with the Union for International Cancer Control, the International Agency for Research on Cancer has also proposed the utilisation of essential TNM that will facilitate the collection of stage data in population-based cancer registries, improve international stage comparisons, and help to elucidate the causes of international variation in survival [46].

Finally, to include the totality of diagnosed cases in all participating jurisdictions and hence increase validity in comparative stage-specific survival, we used multiple imputation to deal with the unknown and missing data for stage at diagnosis. While the degree of stage data completeness varied between jurisdictions (Supplementary Tables 1A and 1B), we observed that the survival for patients with recorded missing stage was between that for patients with stage III and IV tumours (Supplementary Figures $4 \& 5$ ), implying a case mix that is not composed of cases with the most advanced stage only. It is important to note that "unknown" stage does not necessarily imply that clinical stage could not be determined or used for treatment decisions by clinicians at time of diagnosis. Therefore, information on stage may be available from resources other than the registry for cases with "unknown" stage (data missing at random). In such a situation, multiple imputation has been shown to be a valid method for dealing with unknown stage recorded in population-based cancer registry data.[47] After the inclusion of patients whose stage data were imputed, survival estimates were slightly lower in all stages categories, which could be due to the fact that patients with missing data on stage tended to be older and have lower survival.

In conclusion, differences in survival from colon and rectal cancers remain marked across high-income countries in recent years and are more pronounced for older ages and patients with advanced disease. Similarly, the proportions of cases diagnosed with early and advanced colon and rectal cancers differ across countries and survival estimates tended to be lower for countries that had higher proportions of elderly and patients with advanced stage. Our study suggests that both early detection and optimal treatment are important factors that may explain survival gaps between countries. Evidently the improved collection and standardisation of staging data, and the accrual of additional variables such as treatment and comorbidities [48] are critical steps in developing a complete understanding of the underlying mechanisms that explain international differences in cancer survival. 


\section{Acknowledgements}

The authors would like to thank the ICBP management team of Cancer Research UK for managing the programme, the ICBP SurvMark-2 Local Leads for advice to understand the data, for their contributions to the study protocol and interpretation of the results and the ICBP Clinical Committees for their advice. We are also grateful to the ICBP SurvMark-2 Academic Reference Group for providing independent peer review and advice for the study protocol and analysis plan development. Finally we are thankful to the ICBP Programme Board for their oversight and direction. The ICBP is funded by the Canadian Partnership Against Cancer; Cancer Council Victoria; Cancer Institute NSW; Cancer Research UK; Danish Cancer Society; National Cancer Registry Ireland; The Cancer Society of New Zealand; NHS England; Norwegian Cancer Society; Public Health Agency Northern Ireland, on behalf of the Northern Ireland Cancer Registry; The Scottish Government; Western Australia Department of Health; Wales Cancer Network.

Where authors are identified as personnel of the International Agency for Research on Cancer / World Health Organization, the authors alone are responsible for the views expressed in this article and they do not necessarily represent the decisions, policy or views of the International Agency for Research on Cancer / World Health Organization.

Competing interests: The authors declare no relevant competing interests.

\section{Contributorship statement}

Study concept and design: MaA, MeA, FB, IS. Data collection and interpretation of data: HT, LS, RW, NSJ, PD, CMC, GE, AG, EM, PW, CJ, BM, OB. Data analysis: MaA, MJ, AB, JF. Drafting the manuscript: MaA, MeA, IS. Critical revision of the manuscript for important intellectual content: all authors. 


\section{References}

1 Bray F, Ferlay J, Soerjomataram I, Siegel RL, Torre LA, Jemal A. Global cancer statistics 2018: GLOBOCAN estimates of incidence and mortality worldwide for 36 cancers in 185 countries. CA Cancer $\mathrm{J}$ Clin 2018.

2 Allemani C, Matsuda T, Di Carlo V, Harewood R, Matz M, Niksic M, et al. Global surveillance of trends in cancer survival 2000-14 (CONCORD-3): analysis of individual records for 37513025 patients diagnosed with one of 18 cancers from 322 population-based registries in 71 countries. Lancet 2018;391:1023-75.

3 Arnold M, Rutherford MJ, Bardot A, Ferlay J, Andersson TM, Myklebust TA, et al. Progress in cancer survival, mortality, and incidence in seven high-income countries 1995-2014 (ICBP

SURVMARK-2): a population-based study. Lancet Oncol 2019;20:1493-505.

4 Maringe C, Walters S, Rachet B, Butler J, Fields T, Finan P, et al. Stage at diagnosis and colorectal cancer survival in six high-income countries: A population-based study of patients diagnosed during 2000-2007. Acta Oncol 2013;52:919-32.

5 Matsuda T, Yamashita K, Hasegawa H, Oshikiri T, Hosono M, Higashino N, et al. Recent updates in the surgical treatment of colorectal cancer. Ann Gastroenterol Surg 2018;2:129-36.

6 Sargent D, Sobrero A, Grothey A, O'Connell MJ, Buyse M, Andre T, et al. Evidence for cure by adjuvant therapy in colon cancer: observations based on individual patient data from 20,898 patients on 18 randomized trials. J Clin Oncol 2009;27:872-7.

7 Morris EJA, Finan PJ, Spencer K, Geh I, Crellin A, Quirke P, et al. Wide Variation in the Use of Radiotherapy in the Management of Surgically Treated Rectal Cancer Across the English National Health Service. Clin Oncol-Uk 2016;28:522-31.

8 Simmonds PC. Palliative chemotherapy for advanced colorectal cancer: systematic review and meta-analysis. Colorectal Cancer Collaborative Group. BMJ 2000;321:531-5.

9 Van Cutsem E, Cervantes A, Adam R, Sobrero A, Van Krieken JH, Aderka D, et al. ESMO consensus guidelines for the management of patients with metastatic colorectal cancer. Ann Oncol 2016;27:1386-422.

10 Brown S, Castelli M, Hunter DJ, Erskine J, Vedsted P, Foot C, et al. How might healthcare systems influence speed of cancer diagnosis: A narrative review. Soc Sci Med 2014;116:56-63.

11 International Classification of Diseases, Tenth Revision. Epidemiol Bull 1995;16:14-6.

12 Walters S, Maringe C, Butler J, Brierley JD, Rachet B, Coleman MP. Comparability of stage data in cancer registries in six countries: lessons from the International Cancer Benchmarking Partnership. Int J Cancer 2013;132:676-85.

13 Campion WM. Multiple Imputation for Nonresponse in Surveys - Rubin,Db. J Marketing Res 1989;26:485-6.

14 Perme MP, Stare J, Esteve J. On estimation in relative survival. Biometrics 2012;68:113-20.

15 Roche L, Danieli C, Belot A, Grosclaude P, Bouvier AM, Velten M, et al. Cancer net survival on registry data: Use of the new unbiased Pohar-Perme estimator and magnitude of the bias with the classical methods. International Journal of Cancer 2013;132:2359-69.

16 Brenner H, Hakulinen T. Period versus cohort modeling of up-to-date cancer survival. Int J Cancer 2008;122:898-904.

17 Corazziari I, Quinn M, Capocaccia R. Standard cancer patient population for age standardising survival ratios. Eur J Cancer 2004;40:2307-16.

18 Siegel RL, Miller KD, Fedewa SA, Ahnen DJ, Meester RGS, Barzi A, et al. Colorectal cancer statistics, 2017. CA Cancer J Clin 2017;67:177-93.

19 Ciccolallo L, Capocaccia R, Coleman MP, Berrino F, Coebergh JWW, Damhuis RAM, et al. Survival differences between European and US patients with colorectal cancer: role of stage at diagnosis and surgery. Gut 2005;54:268-73. 
20 Schreuders EH, Ruco A, Rabeneck L, Schoen RE, Sung JJ, Young GP, et al. Colorectal cancer screening: a global overview of existing programmes. Gut 2015;64:1637-49.

21 Ministry of Health New Zealand. https://www.health.govt.nz/our-work/preventative-healthwellness/screening/national-bowel-screening-programme Access Date: 23/10/2018.

22 Issa IA, Noureddine M. Colorectal cancer screening: An updated review of the available options. World J Gastroenterol 2017;23:5086-96.

23 Geraghty J, Shawihdi M, Devonport E, Sarkar S, Pearson MG, Bodger K. Reduced risk of emergency admission for colorectal cancer associated with the introduction of bowel cancer screening across England: a retrospective national cohort study. Colorectal Dis 2018;20:94-104.

24 Senore C, Basu P, Anttila A, Ponti A, Tomatis M, Vale DB, et al. Performance of colorectal cancer screening in the European Union Member States: data from the second European screening report. Gut 2018.

25 George S, Primrose J, Talbot R, Smith J, Mullee M, Bailey D, et al. Will Rogers revisited: prospective observational study of survival of 3592 patients with colorectal cancer according to number of nodes examined by pathologists. Br J Cancer 2006;95:841-7.

26 Feinstein AR, Sosin DM, Wells CK. The Will Rogers phenomenon. Stage migration and new diagnostic techniques as a source of misleading statistics for survival in cancer. N Engl J Med 1985;312:1604-8.

27 Weller D, Menon U, Falborg AZ, Jensen H, Barisic A, Knudsen AK, et al. Diagnostic routes and time intervals for patients with colorectal cancer in 10 international jurisdictions; findings from a crosssectional study from the International Cancer Benchmarking Partnership (ICBP). Bmj Open 2018;8. 28 Eden M, Harrison S, Griffin M, Lambe M, Pettersson D, Gavin A, et al. Impact of variation in cancer registration practice on observed international cancer survival differences between International Cancer Benchmarking Partnership (ICBP) jurisdictions. Cancer Epidemiol 2019;58:184-92.

29 Mitchell E, Macdonald S, Campbell NC, Weller D, Macleod U. Influences on pre-hospital delay in the diagnosis of colorectal cancer: a systematic review. Br J Cancer 2008;98:60-70.

30 Forbes LJL, Simon AE, Warburton F, Boniface D, Brain KE, Dessaix A, et al. Differences in cancer awareness and beliefs between Australia, Canada, Denmark, Norway, Sweden and the UK (the International Cancer Benchmarking Partnership): do they contribute to differences in cancer survival? Brit J Cancer 2013;108:292-300.

31 Probst HB, Hussain ZB, Andersen O. Cancer patient pathways in Denmark as a joint effort between bureaucrats, health professionals and politicians--a national Danish project. Health Policy 2012;105:65-70.

32 Torring ML, Frydenberg M, Hamilton W, Hansen RP, Lautrup MD, Vedsted P. Diagnostic interval and mortality in colorectal cancer: U-shaped association demonstrated for three different datasets. J Clin Epidemiol 2012;65:669-78.

33 Toftegaard BS, Guldbrandt LM, Flarup KR, Beyer H, Bro F, Vedsted P. Development of an algorithm to identify urgent referrals for suspected cancer from the Danish Primary Care Referral Database. Clin Epidemiol 2016;8:751-9.

34 Benitez Majano S, Di Girolamo C, Rachet B, Maringe C, Guren MG, Glimelius B, et al. Surgical treatment and survival from colorectal cancer in Denmark, England, Norway, and Sweden: a populationbased study. Lancet Oncol 2019;20:74-87.

35 Schmoll HJ, Van Cutsem E, Stein A, Valentini V, Glimelius B, Haustermans K, et al. ESMO Consensus Guidelines for management of patients with colon and rectal cancer. A personalized approach to clinical decision making. Annals of Oncology 2012;23:2479-516.

36 Babaei M, Balavarca Y, Jansen L, Lemmens V, van Erning FN, van Eycken L, et al. Administration of adjuvant chemotherapy for stage II-III colon cancer patients: An European populationbased study. Int J Cancer 2018;142:1480-9.

37 Glimelius B, Myklebust TA, Lundqvist K, Wibe A, Guren MG. Two countries - Two treatment strategies for rectal cancer. Radiother Oncol 2016;121:357-63. 
38 Allemani C, Rachet B, Weir HK, Richardson LC, Lepage C, Faivre J, et al. Colorectal cancer survival in the USA and Europe: a CONCORD high-resolution study. Bmj Open 2013;3:e003055.

39 Sorbye H, Cvancarova M, Qvortrup C, Pfeiffer P, Glimelius B. Age-dependent improvement in median and long-term survival in unselected population-based Nordic registries of patients with synchronous metastatic colorectal cancer. Ann Oncol 2013;24:2354-60.

40 Parkin DM, Bray F. Evaluation of data quality in the cancer registry: principles and methods Part II. Completeness. Eur J Cancer 2009;45:756-64.

41 Bray F, Parkin DM. Evaluation of data quality in the cancer registry: principles and methods. Part I: comparability, validity and timeliness. Eur J Cancer 2009;45:747-55.

42 Woods LM, Coleman MP, Lawrence G, Rashbass J, Berrino F, Rachet B. Evidence against the proposition that "UK cancer survival statistics are misleading": simulation study with National Cancer Registry data. Bmj-Brit Med J 2011;342.

43 Moller H, Richards S, Hanchett N, Riaz SP, Luchtenborg M, Holmberg L, et al. Completeness of case ascertainment and survival time error in English cancer registries: impact on 1-year survival estimates. Br J Cancer 2011;105:170-6.

44 Eden M, Rous BA, Rashbass J. Misinterpretation of the origins and composition of staging data and its impact on colorectal cancer survival. Acta Oncol 2014;53:845-6.

45 Asli LM, Johannesen TB, Myklebust TA, Moller B, Eriksen MT, Guren MG. Preoperative chemoradiotherapy for rectal cancer and impact on outcomes - A population-based study. Radiother Oncol 2017;123:446-53.

46 Pineros M, Parkin DM, Ward K, Chokunonga E, Ervik M, Farrugia H, et al. Essential TNM: a registry tool to reduce gaps in cancer staging information. Lancet Oncol 2019;20:e103-e11.

47 Nur U, Shack LG, Rachet B, Carpenter JR, Coleman MP. Modelling relative survival in the presence of incomplete data: a tutorial. Int J Epidemiol 2010;39:118-28.

48 Luchtenborg M, Morris EJA, Tataru D, Coupland VH, Smith A, Milne RL, et al. Investigation of the international comparability of population-based routine hospital data set derived comorbidity scores for patients with lung cancer. Thorax 2018;73:339-49. 